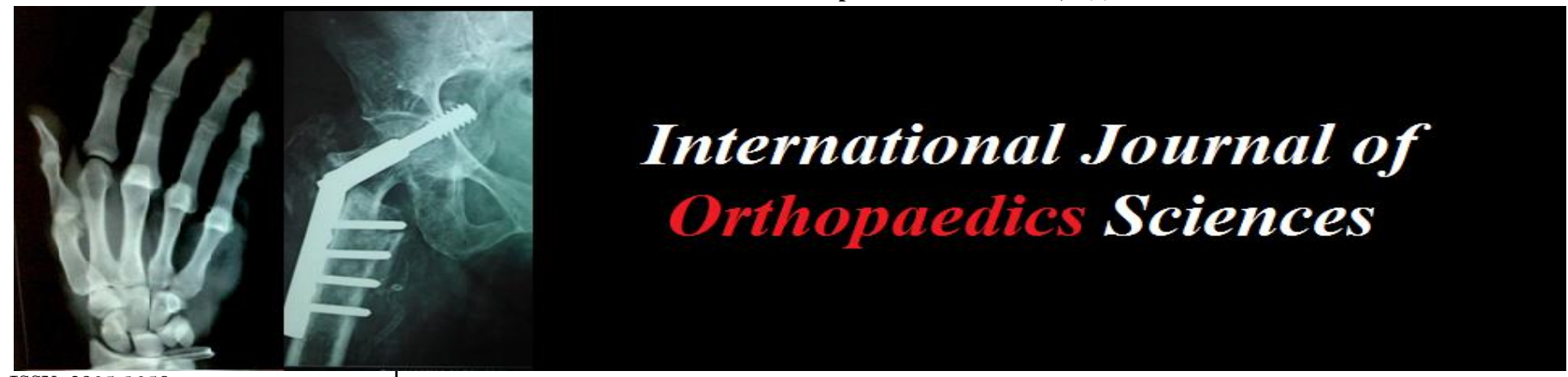

ISSN: 2395-1958

IJOS 2019; 5(1): 507-511

(C) 2019 IJOS

www.orthopaper.com

Received: 26-11-2018

Accepted: 17-12-2018

Amith Sridhar Alankar MS Ortho, AJ Institute of Medical Sciences and Research Centre, Mangalore, Karnataka, India

Sriharsha B

MS Ortho, P.E.S. Institute of Medical Sciences and Research, Kuppam, Karnataka, India

Sachin P Angadi

D Ortho Sri Devaraj Urs Medical College, Kolar, Karnataka, India

D Ayush Sidde Gowda MBBS, St. Philomena's Hospital, Bangalore, Karnataka, India
Correspondence

Amith Sridhar Alankar MS Ortho, AJ Institute of Medical Sciences and Research Centre, Mangalore, Karnataka, India

\section{Functional outcome following arthroscopic anterior cruciate ligament reconstruction using quadrupled hamstring autograft}

\author{
Amith Sridhar Alankar, Sriharsha B, Sachin P Angadi and D Ayush \\ Sidde Gowda
}

DOI: https://doi.org/10.22271/ortho.2019.v5.i1i.90

Abstract

Background: Arthroscopic reconstruction of ACL is a well-accepted treatment of ACL injuries; however, no uniform consensus exists to decide type of fixation for arthroscopic anterior cruciate ligament reconstruction

Materials and Methods: A total of 30 patients underwent arthroscopic anterior cruciate ligament reconstruction with hamstring tendons using endobutton on femoral side and interference screw on tibial side. The evaluation methods were clinical examination, Lysholm and IKDC score. Patients were followed up at the end of 1 month, 3 months, 6 months and 8 months and their functional outcome was assessed

Results: In our study of sample size 30 with male preponderance $(93.33 \%)$. The mean age was 26.46 years. Mean post-operative Lysholm score was 87.95 , mean post-operative IKDC score was 79.88 at the end of 8 months. 25 patients $(83.3 \%)$ were able to return to their pre-injury activity.

Conclusion: Arthroscopic anterior cruciate ligament reconstruction with hamstring tendons using endobutton and interference screw had good functional outcome.

Keywords: ACL, arthroscopy, hamstring graft, endobutton, knee injury

\section{Introduction}

ACL is the primary $(85 \%)$ restraint to limit anterior translation of the tibia. The tensile strength of ACL is $2160 \mathrm{~N}$. Incidence being 1 in 3,000 amongst the general population ${ }^{[1]}$. They are common among sports injuries caused by brutal deceleration movements.

Galen ${ }^{[2]}$ was the first to describe the ACL as being a structure that supports the joint and prevents abnormal knee motion. George K Noulis ${ }^{[3]}$ precisely described the function of the ACL, in the year of 1875, in his thesis on "Knee sprains". He also stated that the integrity of the ligament should be assessed in knee extension. The test which was put forth by Noulis is identified to the one which is now known and used as the Lachman test, which is the most sensitive test for ACL tears. A.W. Mayo Robson ${ }^{[4]}$ in 1895, executed the first ACL repair. ACL reconstruction has remained a treatment of choice for anterior cruciate ligament deficient knees, since majority of non-operative procedures have resulted in functionally unacceptable outcomes.

\section{Anatomy}

The ACL is a ligamentous structure composed of dense connective tissue, made of parallel rows of type 1 collagen and fibroblast ${ }^{[5]}$. It originates from the posterior and medial aspect of the lateral femoral condyle and inserts to the anterior and medial aspect of the medial tibial spine ${ }^{[6]}$.

The ACL is composed of two principal parts: 1. Small anteromedial bundle (AMB) 2. Large bulky posterolateral bundle (PLB). During extension of knee, PLB tightens and AMB relaxes, however during flexion of knee, AMB tightens and PLB relaxes, hence producing a twist and untwist motion. 

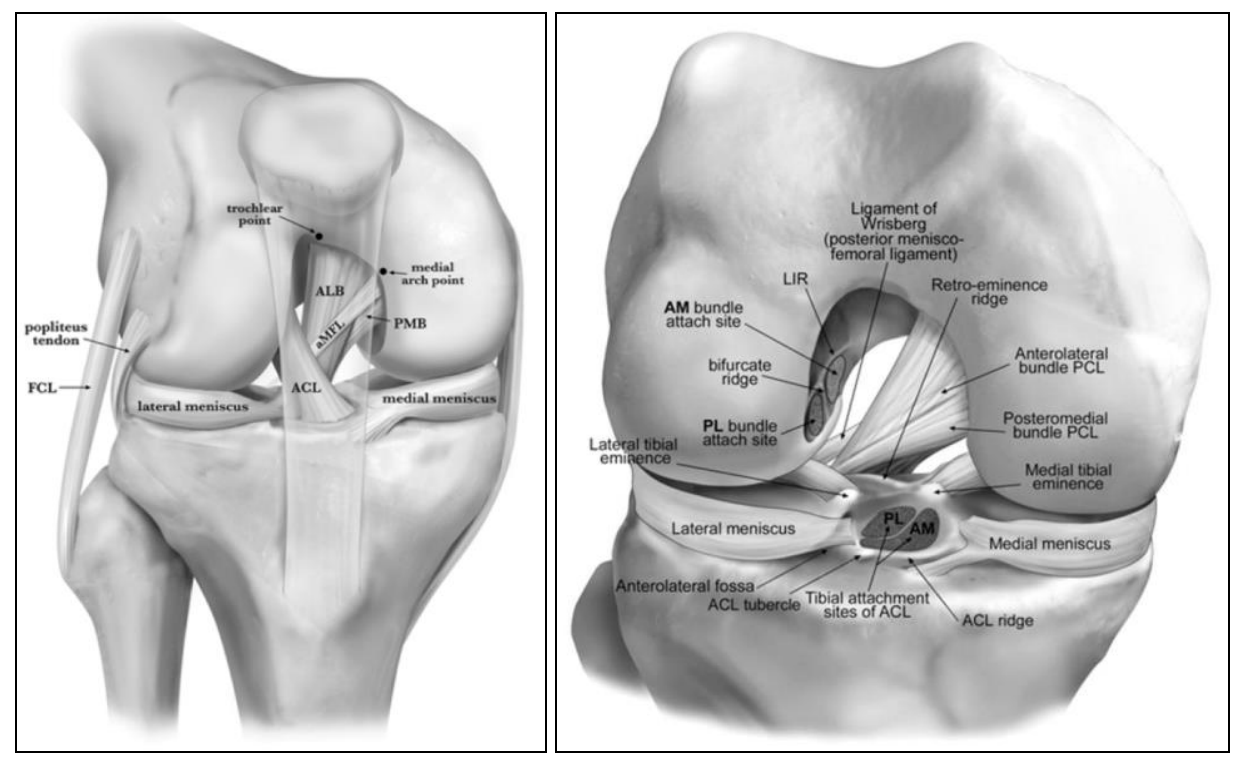

Fig 1: Anatomy of the ACL showing AMB and PLB foot prints on femoral and tibial ends

\section{Objective}

To evaluate the functional outcome of arthroscopic anterior cruciate ligament reconstruction using semitendinosus and gracilis autograft and to assess complications following the reconstruction.

\section{Materials and Methods}

This is a prospective cohort study of 30 consecutive patients who underwent arthroscopic ACL reconstruction using quadrupled semitendinosus tendon autograft during the study period, July 2016 to September 2018, in AJ Institute of Medical Sciences and Research Centre, Mangalore after obtaining ethical committee clearance.

Inclusion criteria

- Anterior cruciate ligament tear confirmed either clinically or radiologically by MRI.

- $\quad$ Age between $18-45$ years

\section{Exclusion criteria}

- Anterior cruciate ligament tear associated with bony injury around the knee.

- Any previously operated knees for any reason

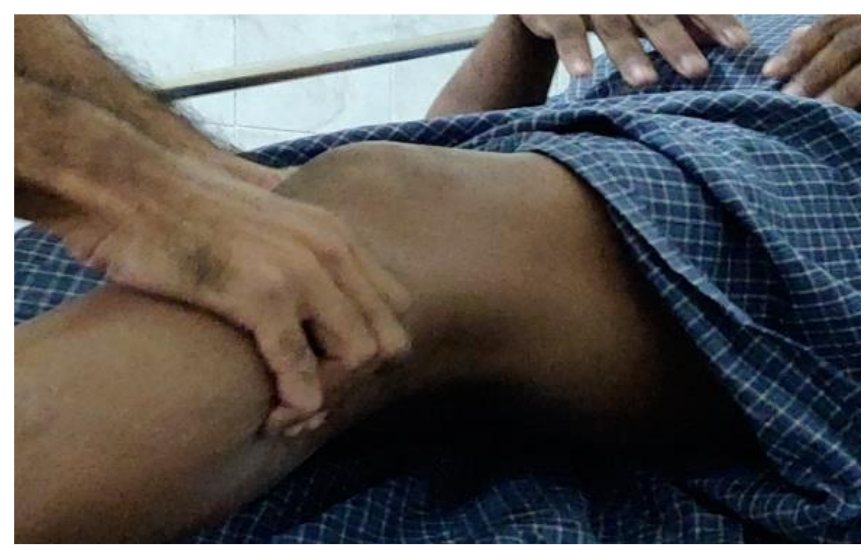

Fig 2: Lachman Test

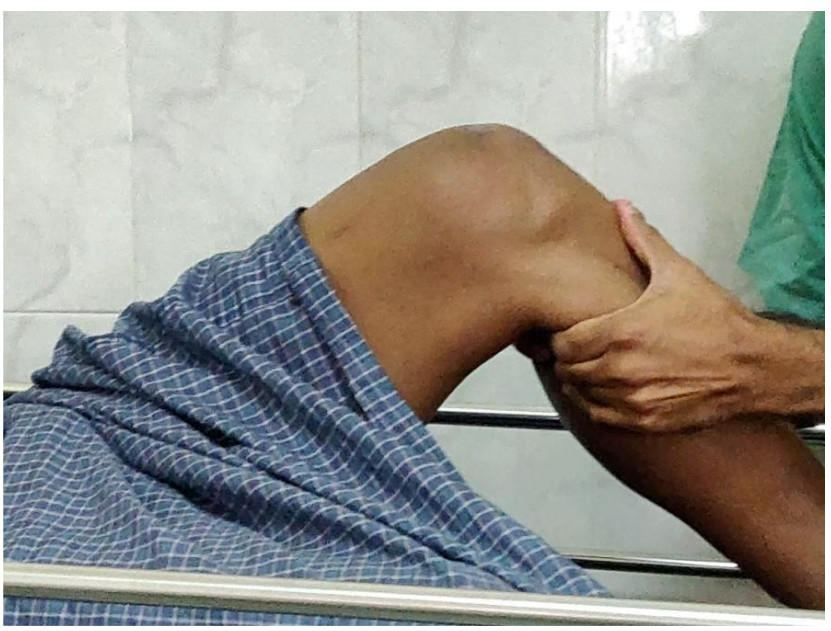

Fig 3: Anterior drawer test

\section{Surgical Technique}

All the surgical procedures were performed by the same surgeon. Tourniquet was used in all cases. Standard anteromedial (AM) and anterolateral (AL) portals were used. Diagnostic arthroscopy was performed, and ACL tear confirmed. A $3-4 \mathrm{~cm}$ incision anteromedially on the tibia to expose and harvest the hamstring tendons (semitendinosus and gracilis tendons). Graft quadrupled and prepared. Femoral tunnel and tibial tunnel prepared. Endobutton on to femoral side and interference screw on tibial side.

Post-operative protocol was standard for all the patients. Static quadriceps strengthening exercises, active knee bending with gradual increase of $10-20^{\circ}$ of flexion per day, assisted SLRT, abduction and adduction exercises of thigh, hamstring strengthening exercises were started from day 1 to 7 . Walking with knee brace by the end of $1^{\text {st }}$ week. Closed kinetic chain exercises from 3-6 weeks. Ellipticals, treadmill walking from 6-12 weeks. Forward running, ladder drills, agility training from 3-6 months. 


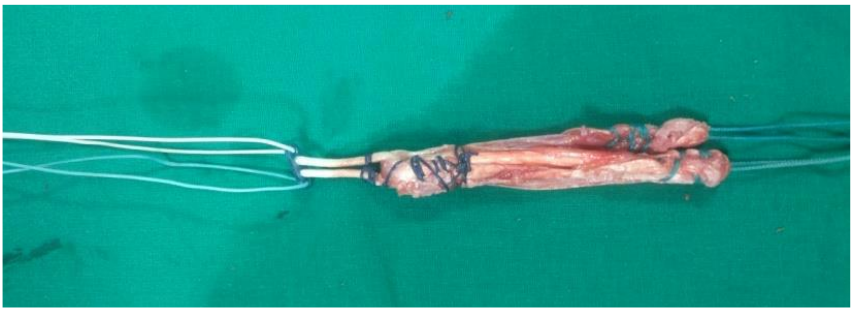

Fig 4: prepared and quadrupled hamstring graft

\section{Statistical analysis}

Descriptive statistics are reported (mean, median, minimum, maximum, standard deviation). Descriptive statistics like mean, standard deviation was computed for continuous variables and frequency and proportions for categorical data Association between two variables were found using Chi square test. Independent $t$ test was done to compare the group means. P-value $<0.05$ was considered as statistically significant. The STATA 14.0 software was used. Microsoft Word and Excel were used to generate graphs and tables.

\section{Results}

The mean age in our study was 26.46 years. The maximum number of patients were in the age group of 21- 25yrs $(46.6 \%)$. Out of 30 patients, 28 patients $(93.33 \%)$ were males and 2 patient $(6.67 \%)$ females. Right knee was injured in 19 patients $(63 \%)$ and left knee in 11 patients $(37 \%)$. Majority of the patients $(46.6 \%)$ were from agricultural occupation followed by sports $(33.3 \%)$ and $20 \%$ were into sedentary lifestyle.

Table 1: Distribution based on occupation of the patients

\begin{tabular}{|c|c|c|}
\hline Occupation & Frequency $(\mathbf{n}=\mathbf{3 0})$ & Percentage \\
\hline Agriculture & 14 & $46.6 \%$ \\
\hline Sports & 10 & $33.3 \%$ \\
\hline Sedentary & 6 & $20 \%$ \\
\hline
\end{tabular}

Medial meniscal tear was the commonest associated injury (33.3\%) detected by MRI followed by lateral meniscus $(26.6 \%)$ and medial collateral ligament injury (6.6\%). There was no lateral collateral ligament and PCL injury.
Table 2: Distribution of associated injuries

\begin{tabular}{|c|c|c|}
\hline & Frequency $(\mathbf{n = 3 0})$ & Percentage \\
\hline MM & 10 & $33.3 \%$ \\
\hline LM & 8 & $26.6 \%$ \\
\hline MCL & 2 & $6.6 \%$ \\
\hline LCL & 0 & $0 \%$ \\
\hline PCL & 0 & $0 \%$ \\
\hline
\end{tabular}

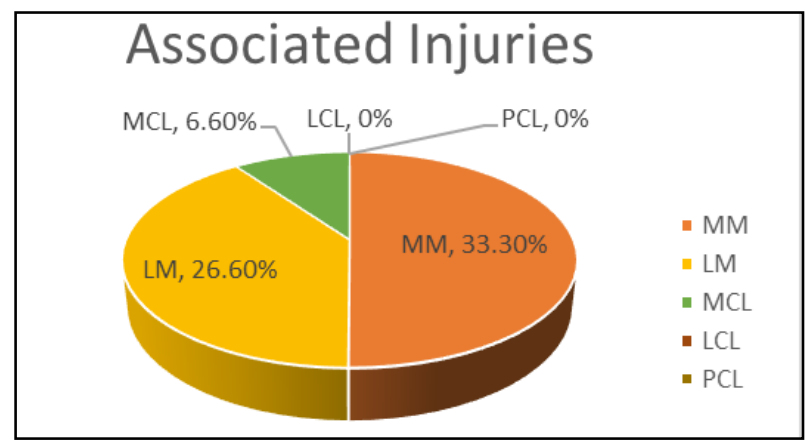

Fig 5: Distribution of associated injuries

6 patients $(20 \%)$ had pain at the graft site at the end of 6 months. Early superficial infection of the site was present in 1 case $(3.3 \%)$ with delayed wound healing and regaining full function. 14 patients $(46.6 \%)$ had grade I laxity at the end of 6 months but with hard end point. No patients had deep infection and FFD following the surgery.

Table 3: Complications

\begin{tabular}{|c|c|c|}
\hline & Frequency $(\mathbf{n}=\mathbf{3 0})$ & Percentage \\
\hline Pain & 6 & $20 \%$ \\
\hline Superficial Infection & 1 & $3.3 \%$ \\
\hline Deep Infection & 0 & $0 \%$ \\
\hline Numbness & 4 & $13.3 \%$ \\
\hline Laxity & 14 & $46.6 \%$ \\
\hline Click & 2 & $6.6 \%$ \\
\hline FFD & 0 & $0 \%$ \\
\hline
\end{tabular}

Table 4: Pre-operative Scores

\begin{tabular}{|c|c|}
\hline IKDC & Lysholm \\
\hline $54.34367+/-3.656798$ & $64.73333+/-4.266415$ \\
\hline
\end{tabular}

Table 5: Postoperative scoring and outcome

\begin{tabular}{|c|c|c|c|c|c|c|c|}
\hline & IKDC & min & max & Lysholm & min & max & p value \\
\hline 1 month & 62.40533 & 52 & 78.16 & 72.33333 & 64 & 80 & \\
\cline { 1 - 8 } 3 months & 69.66667 & 58 & 86 & 80.2 & 69 & 87 & \multirow{2}{*}{$<0.05$} \\
\hline 6 months & 76.27 & 64 & 93.1 & 85.93333 & 72 & 92 & \\
\hline 8 months & 79.86667 & 70 & 96.6 & 87.95 & 76 & 94 & \\
\hline
\end{tabular}

According to IKDC score, $90 \%$ of the patients graded their post-operative recovery as normal and $30 \%$ as near normal whereas 3 patients (10\%) graded recovery as abnormal.

According to Lysholm scores, 7 patients $(23.33 \%)$ fell under excellent recovery group. 20 patients $(66.66 \%)$ had good postoperative outcome. 3 patients $(10 \%)$ had a fair outcome. and no patients had poor outcome. Majority of patients fell in 8490 category (good outcome).

Table 6: Post-operative grading according to IKDC

\begin{tabular}{|c|c|c|}
\hline Score Category & Frequency $(\mathbf{n}=\mathbf{3 0})$ & Percentage $\mathbf{( \% )}$ \\
\hline Excellent $(91-100)$ & 7 & 23.33 \\
\hline Good $(84-90)$ & 20 & 66.66 \\
\hline Fair $(65-83)$ & 3 & 10 \\
\hline Poor $(<65)$ & 0 & 0 \\
\hline
\end{tabular}

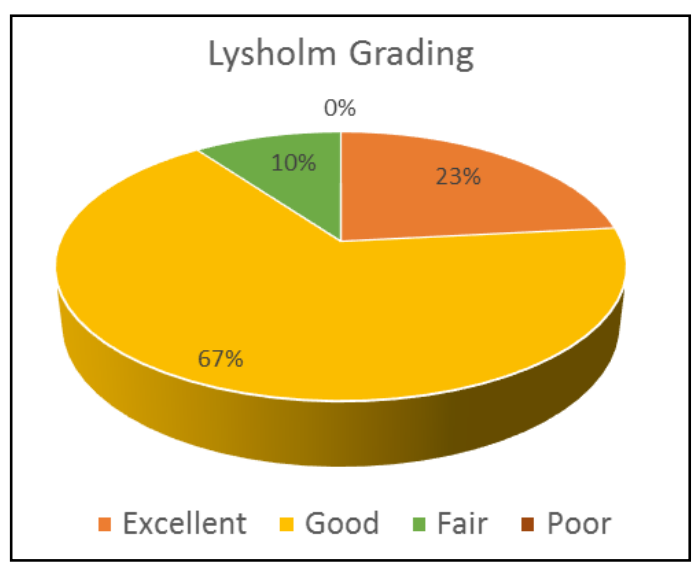

Fig 6: Post-operative distribution according to Lysholm 
25 patients $(83.3 \%)$ were able to return to their pre-injury activity including farming and to competitive sports. 5 patients $(16.6 \%)$ were not satisfied with physiotherapy regimen and these patients were noncompliant to the protocol at the last months. But regained full flexion and could do daily activities.
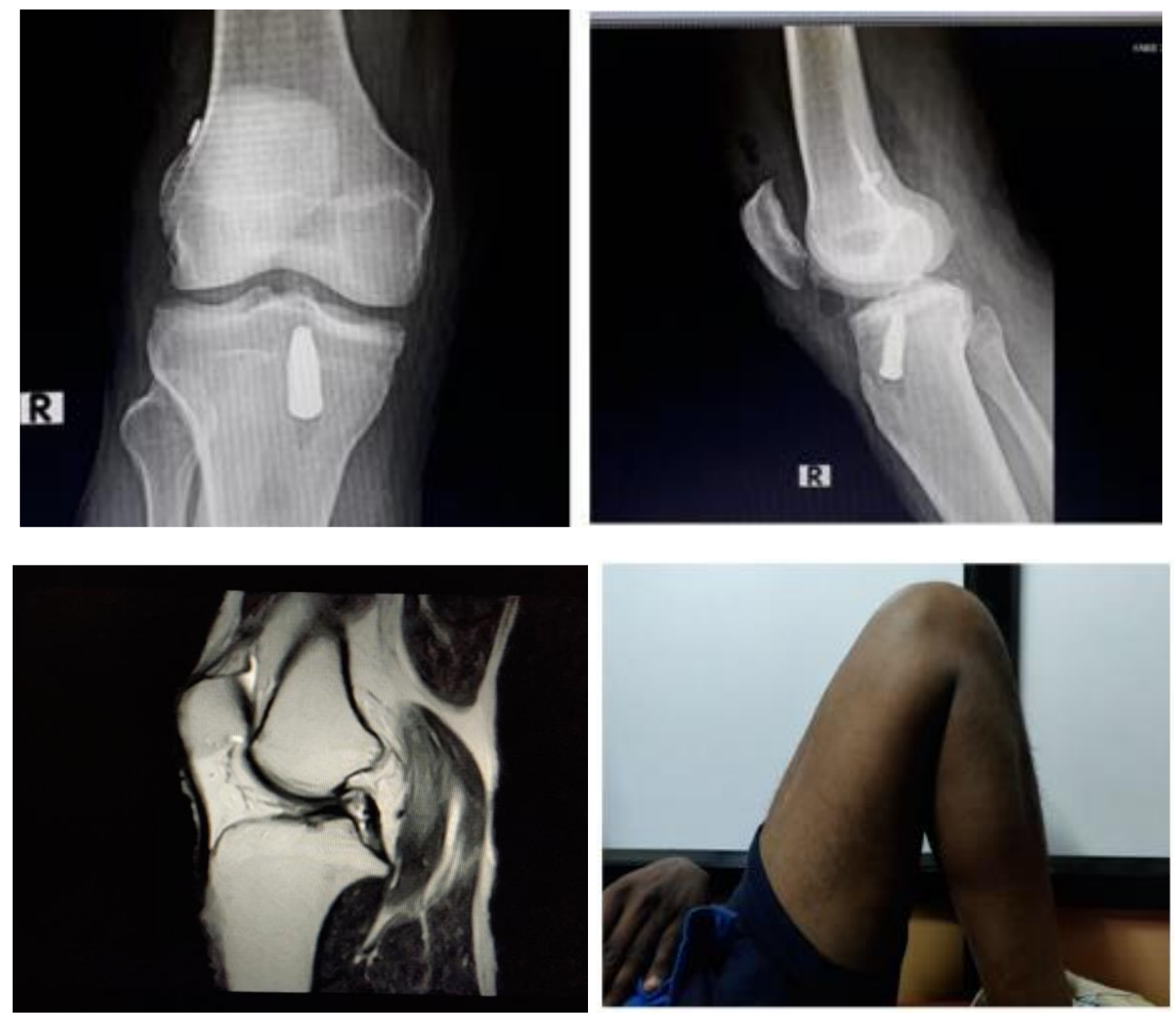

Fig 7: A 27-year-old male patient with right ACL tear confirmed with MRI, achieved complete knee flexion following 8 months of arthroscopic reconstruction

\section{Discussion}

Anterior cruciate ligament (ACL) ruptures left untreated lead to subsequent knee disability, which can be severe with potentially devastating long-term consequences. With improving results and increasingly reliable outcomes, patient and physician expectations have evolved to include the goal of return to activities and sports at normal or near normal levels.

In 2009, Brown ${ }^{[7]}$ and others studied the incidence of sex and limb differences in anterior cruciate ligament injury and stated that even though females are prone for injury, due to their less exposure to strenuous environment makes the incidence of males more than females. They also concluded that limb differences have no influence either during injury or in the recovery period.

In our study Medial meniscal tear was the commonest associated injury (33.3\%) detected by MRI followed by lateral meniscus $(26.6 \%)$. Vassilios S Nikolaou et al. ${ }^{[8]}$ in 2008, after a retrospective analysis of MRI efficiency in diagnosing internal lesions of the knee, reported that the accuracy for tears to the medial, lateral meniscus, anterior and posterior cruciate ligaments and articular cartilage was $81 \%$, $77 \%, 86 \%, 98 \%$ and $60 \%$ respectively. Williams et al. ${ }^{[9]}$ in their study of 2500 cases of arthroscopic ACL reconstruction, reported an infection rate of $0.3 \%$. In our study, one patient had superficial infection. The patient with superficial infection was managed with antibiotics alone and had good functional outcome at the end of 6 months.

Nebelung et al. ${ }^{[10]}$ reviewed the results of twenty-nine anterior cruciate reconstructions with autogenous semitendinosus tendon and a femoral Endobutton. They graded $66 \%$ of the results as normal or nearly normal using the criteria of the IKDC. The Lysholm score improved from a preoperative mean (and standard deviation) of $55 \pm 3$ points (range, 15 to 100 points) to a postoperative mean of $91 \pm 2$ points (range, 55 to 100 points) ( $<<0.01$ ) as observed by Williams III RJ et al. ${ }^{[11]}$ in 2004. Colombet, $\mathrm{P}$ et al. ${ }^{[12]}$ in their study observed that IKDC subjective score Preoperative was $60.4 \pm 15.0$ (33.3 to 95.4) and 2-year postoperative improved to $87.6 \pm 10.6$ (43.7 to 100.0). Lysholm score Preoperative was $75.6 \pm 13.0$ (44.0 to 100.0) 2-year postoperative was $90.8 \pm 9.3$ (56.0 to 100.0$)$ which is comparable with our study.

\section{Conclusion}

- In young active adults, anatomic single bundle reconstruction with quadrupled hamstring graft gives good functional results

- The absence of patellofemoral pain with the use of hamstring graft makes it a more desirable option for patients with patellofemoral cartilage disorders or those with chronic patellofemoral pain

- Hamstring graft fixation with endobutton and 
interference screw gives good functional outcome

- Arthroscopic anterior cruciate ligament reconstruction with hamstring graft is an excellent treatment option for anterior cruciate ligament deficient knees

\section{Limitations}

- Small sample size.

- The results of the study were assessed using subjective scores and not based on objective assessment.

- Short duration of follow up.

\section{References}

1. Lyman S, Koulouvaris P, Sherman S, Do H, Mandl LA, Marx RG. Epidemiology of anterior cruciate ligament reconstruction: trends, readmissions, and subsequent knee surgery. J Bone Joint Surg Am. 2009; 91(10):2321-8.

2. Galen C. On the usefulness of the parts of the body. Ithaca Cotnett University Press, 1968.

3. Segond, Paul-Ferdinand (1851-1912) - Clinical and experimental research on sprained knee blood effusions; Medical Progress, 1879 (Paris)

4. Mayo Robson AW. - Ruptured cruciate ligaments and their repair by operation. Ann Surg. 1903; 37:716-718.

5. Amiel D, Frank C, Harwood F et al Tendons and ligaments: a morphological and biochemical comparison. J Orthop Res. 1984; 1:257-265

6. Petersen W, Tillmann B. Anatomy and function of the anterior cruciate ligament. Orthopäde. 2002; 31:710-718 Duthon VB, Barea C, Abrassart S et al (2006) Anatomy of the anterior cruciate ligament. Knee Surg Sports TraumatolArthrosc 14:204-213; Harner CD, Baek GH, Vogrin TM et al (1999) Quantitative analysis of anterior cruciate ligament insertions. Arthroscopy 15:741-749

7. Brown TN, Palmieri Smith RM, Mclean SG. Sex and limb differences in hip and knee kinematics and kinetics during anticipated and unanticipated jump landings: implications for anterior cruciate ligament injury. $\mathrm{Br} \mathrm{J}$ Sports Med. 2009; 43:1049- 56

8. Nikolaou VS, Chromopoulos E, Savvidou C, Plessas S, Giannoudis $\mathrm{P}$, Nicolas $\mathrm{E}$ et al,. MRI efficacy in diagnosing internal lesions of knee: a retrospective analysis. J Trauma Management \& Outcomes 2008, 0204.

9. Williams RJ, 3rd, Laurencin CT, Warren RF, Speciale AC, Brause BD, O'Brien S. Septic arthritis after arthroscopic anterior cruciate ligament reconstruction. Diagnosis and management. Am J Sports Med. 1997; 25:261-7.

10. Nebelung W, Becker R, Merkel M, Ropke M. Bone tunnel enlargement after anterior cruciate ligament reconstruction with semitendinosus tendon using Endobutton fixation on the femoral side. Arthroscopy. 1998; 14:810-5.

11. Williams III RJ, Hyman J, Petrigliano F, Rozental T, Wickiewicz TL. Anterior cruciate ligament reconstruction with a four-strand hamstring tendon autograft. JBJS. 2004; 86(2):225-32.

12. Colombet P, Saffarini M, Bouguennec N. Clinical and Functional Outcomes of Anterior Cruciate Ligament Reconstruction at a Minimum of 2 Years Using Adjustable Suspensory Fixation in Both the Femur and Tibia: A Prospective Study. Orthopaedic journal of sports medicine. 2018; 6(10):2325967118804128. doi:10.1177/2325967118804128 